Eduardo Carvalho Pessoa ${ }^{1}$

Jose RicARDo PaciêncIa RodRIGUES ${ }^{2}$

ODAIR MICHELIN ${ }^{3}$

Heloisa Vespolu de LUCA

Carla Prisclla Kamita ${ }^{5}$

Paulo Traiman ${ }^{6}$

Gilberto Uemura ${ }^{\top}$

Artigos originais

Palavras-chaves

Neoplasias mamárias/quimioterapia Terapia neoadjuvante

Estudos retrospectivos

Keywords

Breast neoplasm/drug

Therapy

Neoadjuvant therapy

Retrospective studies

\section{Avaliação da resposta à quimioterapia primária em amostra de mulheres brasileiras com tumores de mama localmente avançados}

\author{
Evaluation of response to primary chemotherapy in Brazilian \\ patients with locally advanced breast cancer
}

Resumo

OBJETIVO: avaliar a resposta loco-regional à quimioterapia primária nas pacientes com câncer de mama nos estadios II e III. MÉTODOS: foi realizado um estudo clínico retrospectivo e analíitico de 97 pacientes no estadios II e III, no período de janeiro de 1993 a dezembro de 2004, submetidas a três ou quatro ciclos de quimioterapia primária com 5-fluorouracil $\left(500 \mathrm{mg} / \mathrm{m}^{2}\right)$, epirrubicina $\left(50 \mathrm{mg} / \mathrm{m}^{2}\right)$ e ciclofosfamida $\left(500 \mathrm{mg} / \mathrm{m}^{2}\right)$ ou doxorrubicina $\left(50 \mathrm{mg} / \mathrm{m}^{2}\right)$ e ciclofosfamida $\left(500 \mathrm{mg} / \mathrm{m}^{2}\right)$ e posteriormente ao tratamento loco-regional cirúrgico conservador ou radical. Para estudo da associação entre as variáveis (idade, estado menopausal, volume tumoral pré-quimioterapia, estado axilar, estádio, esquema terapêutico e número de ciclos) foram utilizados os testes do $\chi^{2}$ e o exato de Fisher. Para as variáveis quantitativas (volume tumoral pelo estudo anátomo-patológico e volume tumoral clínico pós-quimioterapia) foi utilizado o coeficiente de correlação de Pearson. O nível de significância utilizado foi de 5\%. RESULTADOS: a média de idade da população estudada foi de 52,2 anos. No estádio II, tivemos 56,8\% dos casos e no estádio III, 43,2\%. Aproximadamente metade das pacientes receberam FEC50 e 50\%, AC. Obtivemos uma resposta clínica objetiva com o tratamento quimioterápico primário em $64,9 \%$ dos casos. A resposta clínica completa ocorrev em $12,3 \%$ das pacientes; iá a resposta patológica completa aconteceu em 10,3\% dos casos. Observamos uma correlação significante entre o número de ciclos e a resposta à quimioterapia primária. Também verificamos uma concordância significante entre a avaliação pelo exame clínico da resposta à quimioterapia primária e o achado anátomo-patológico. CONCLUSÕES: o número de ciclos foi importante para a resposta loco-regional, sendo que as pacientes que receberam maior número de ciclos obtiveram melhores respostas. Também foi possível avaliar a resposta tumoral pelo exame clínico, pois houve concordância com o anátomo-patológico.

\section{Abstract}

PURPOSE: to evaluate the loco-regional response to primary chemotherapy in patients with breast cancer at stages II and III. METHODS: a retrospective and analytical clinical study carried out in 97 patients with an average age of 52.2 years old, with breast cancer at stages II and III, attended from January 1993 to December 2004, and submitted to 3 to 4 cycles of primary chemotherapy with 5 -fluorouracil $-500 \mathrm{mg} / \mathrm{m} 2$, epirubicin $-50 \mathrm{mg} / \mathrm{m} 2$ and cyclophosphamide $-500 \mathrm{mg} / \mathrm{m} 2$ or doxorubicin $-50 \mathrm{mg} / \mathrm{m} 2$ e cyclophosphamide $-500 \mathrm{mg} / \mathrm{m} 2$, and then to loco-regional surgical conservative or radical surgical treatment. Chi-square and Fisher's exact tests were used to study the association among the variables (age, menopausal state, pre-chemotherapy tumoral volume, axillary condition, stage, therapeutic scheme and number of cycles), while Pearson's correlation coefficient was used for the quantitative variables (tumoral volume according to the anatomo-pathological study and the post-chemotherapy clinical tumoral volume. The significance level was $5 \%$. RESULTS: there were $56.8 \%$ of cases at stage II and $43.2 \%$ at stage III. Approximately $50 \%$ of the patients received FEC50 and 50\% AC. Objective clinical response with primary chemotherapy was obtained in $64.9 \%$ of the cases. Full clinical response occurred in $12.3 \%$ of patients, while full pathological response occurred in $10.3 \%$ of the
Correspondência:

Eduardo Carvalho Pessoa Departamento de Ginecologia e Obstetríicia da Faculdade de Medicina da Universidade Estadual Paulista "Júlio Mesquita Filho" - UNESP - Botucatu (SP), Brasil. Distrito Rubião Júnior, $s / n^{0}$ - CEP 18618-970 - Botucatu/SP Fone: (14) 3811-6528/ (14) 9776-0333 E-mail: carvalho-pesson@uol.com.br

Recebido

14/02/2006

Aceito com modificacões

$21 / 12 / 2006$
Trabalho realizado no Centro de Avaliação em Mastologia "Prof. Laurival Antonio De Luca" do Departamento de Ginecologia, Obstetrícia e Mastologia da Faculdade de Medicina da Universidade Estadual Paulista "Júlio Mesquita Filho" - UNESP - Botucatu (SP), Brasil.

'Médico do Departamento de Ginecologia, Obstetrícia e Mastologia da Faculdade de Medicina da Universidade Estadual Paulista "Júlio Mesquita Filho" - UNESP - Botucatu (SP), Brasil.

Professor do Departamento de Ginecologia, Obstetrícia e Mastologia da Faculdade de Medicina da Universidade Estadual Paulista "Júlio Mesquita Filho" - UNESP - Botucatu (SP), Brasil.

Professor do Departamento de Clínica Médica da Faculdade de Medicina da Universidade Estadual Paulista "Júlio Mesquita Filho" - UNESP - Botucatu (SP), Brasil.

${ }^{4}$ Médica do Departamento de Ginecologia, Obstetrícia e Mastologia da Faculdade de Medicina da Universidade Estadual Paulista "Júlio Mesquita Filho" - UNESP - Botucatu (SP), Brasil.

${ }_{5}^{5}$ Médica do Centro de Avaliação e Mastologia da Faculdade de Medicina da Universidade Estadual Paulista "Júlio Mesquita Filho" - UNESP - Botucatu (SP), Brasil.

- Professor Adjunto do Departamento de Ginecologia, Obstetrícia e Mastologia da Faculdade de Medicina da Universidade Estadual Paulista "Júlio Mesquita Filho" - UNESP - Botucatu (SP), Brasil.

Professor do Departamento de Ginecologia, Obstetrícia e Mastologia da Faculdade de Medicina da Universidade Estadual Paulista "Júlio Mesquita Filho" - UNESP - Botucatu (SP), Brasil. 
cases. CONCLUSIONS: there was a statistically significant correlation between the number of cycles and the response to primary chemotherapy. Patients who received 4 cycles had better response than those who received 3 cycles. There was also a statistically significant concordance between the evaluation through clinical examination of the response to primary chemotherapy and the pathological findings. No statistically significant correlation was observed concerning age, menopausal status, tumoral volume, and pretreatment of axillary damage.

\section{Introdução}

A quimioterapia neoadjuvante, também conhecida como primária, pré-operatória ou de indução, tem conquistado um papel promissor no tratamento do câncer de mama localmente avançado. Este tipo de terapêutica consiste na administração do quimioterápico antes do tratamento cirúrgico. As vantagens práticas e teóricas da quimioterapia primária são: reduzir o tamanho do tumor e aumentar a taxa de cirurgia conservadora da mama ou tornar operáveis tumores localmente avançados; impedir o crescimento dos focos metastáticos após a remoção do tumor primário; diminuir o potencial de clones químio-resistentes; avaliar a sensibilidade tumoral in vivo e permitir o estudo dos marcadores biológicos como, por exemplo, os receptores hormonais ${ }^{1}$.

Um dos mais importantes estudos realizados sobre quimioterapia neoadjuvante é o NSABP B-18, que avaliou 1500 mulheres, divididas em dois grupos. O primeiro recebeu tratamento neoadjuvante com Adriamicina Ciclofosfamida (AC) e o segundo recebeu quimioterapia adjuvante, também com AC. Trinta e seis por cento das pacientes tiveram resposta clínica completa e 13\% resposta patológica completa. Após cinco anos de seguimento, não houve diferença entre os dois braços do estudo tanto na taxa de recidiva local quanto à presença de doença a distância. As pacientes com resposta clínica completa tiveram a mesma sobrevida global, porém, um período livre de doença maior. Nas pacientes com resposta patológica completa observaram-se sobrevida global e período livre de doenças maiores ${ }^{2}$.

Novas drogas, como os taxanes, são atualmente muito empregadas para esta forma de tratamento. Um dos primeiros estudos em que se avaliou a adição dos taxanes aos antracíclicos foi o NSABP-27, que dividiu 2411 mulheres com câncer de mama (T1c-T3, N0-N1, M0) em três grupos. No primeiro grupo, as pacientes receberam quatro ciclos de AC pré-operatórios; no segundo grupo, as pacientes receberam quatro ciclos de AC pré-operatórios seguidos de quatro ciclos de docetaxel; e, no terceiro grupo, as pacientes também receberam quatro ciclos pré-operatórios de AC e quatro ciclos pós-operatórios de docetaxel. Foram observados $45,4 \%$ de resposta clínica parcial, 40,2\% de resposta clínica completa e, aproximadamente, $10 \%$ de resposta patológica completa no primeiro e no terceiro grupo.
Já no grupo que recebeu a adição do docetaxel, a resposta clínica parcial foi de $27,1 \%$, a resposta clínica completa de $63,6 \%$, e a resposta patológica completa de $18,9 \%^{3}$. Uma revisão sistemática sobre o emprego dos taxanes na quimioterapia primária conclui que o seu uso é seguro e eficaz, além de apresentar uma resposta clínica, patológica, sobrevida livre de doença e sobrevida global maiores que as obtidas apenas com o uso de regimes baseados em antracíclicos ${ }^{1}$.

Também há um novo horizonte com o uso de trastuzumab (anticorpo monoclonal com o receptor de membrana HER-2), associado a taxanes e antracíclicos para quimioterapia neoadjuvante. Esquemas com este medicamento têm mostrado altas taxas de resposta patológica completa (até $66,7 \%)^{4}$.

Com particularidade no Brasil aproximadamente metade dos tumores de mama são diagnosticados em estádios III e IV ${ }^{5}$, o que oferece um amplo campo para o uso da quimioterapia primária. A população brasileira, porém, tem suas características peculiares e a grande maioria das mulheres com a neoplasia maligna da mama no Brasil são atendidas através do Sistema Único de Saúde (SUS), que oferece como opção para o tratamento neoadjuvante os regimes baseados em antracíclicos. Assim, tornam-se necessários estudos nacionais que englobem esta realidade. É dentro desta ótica que o nosso trabalho propõe-se a avaliação dos fatores anátomo-clínicos relacionados com a resposta loco-regional à quimioterapia neoadjuvante com regimes baseados nos antracíclicos em pacientes brasileiras portadoras de carcinomas localmente avançados tratados em nosso serviço.

\section{Métodos}

\section{Desenho do estudo}

O presente trabalho constitui-se em estudo clínico, de caráter analítico e retrospectivo, em que se utilizaram os dados dos prontuários de mulheres portadoras de carcinoma da mama, estádios IIA, IIB, IIIA e IIIB, submetidas à quimioterapia primária. As pacientes foram tratadas no Centro de Avaliação em Mastologia "Prof. Laurival Antonio De Luca" do Departamento de Ginecologia, Obstetrícia e Mastologia da Faculdade 
de Medicina de Botucatu da Universidade Estadual Paulista "Júlio de Mesquita Filho" (UNESP), no período de janeiro de 1993 a dezembro de 2004. Foram selecionadas 97 pacientes submetidas a três ou quatro ciclos de quimioterapia primária após o diagnóstico baseado em biópsias percutâneas e que, posteriormente, foram submetidas ao tratamento loco-regional cirúrgico conservador ou radical.

Os critérios de seleção foram: pacientes portadoras de carcinoma de mama nos estádios IIA, IIB, IIIA e IIIB (sistema TNM, adotado pela União Internacional Contra o Câncer, UICC6 $6^{6}$ ), com tumores mensuráveis ao exame clínico e diagnosticados por biópsia de agulha grossa, cujo tratamento inicial foi a quimioterapia, no total de três a quatro ciclos, com o seguinte esquema de drogas: doxorubicina $\left(60 \mathrm{mg} / \mathrm{m}^{2}\right)$ e ciclofosfamida $\left(600 \mathrm{mg} / \mathrm{m}^{2}\right)(\mathrm{AC})$ ou 5 -fluorouracil $\left(500 \mathrm{mg} / \mathrm{m}^{2}\right)$, epirubicina $\left(50 \mathrm{mg} / \mathrm{m}^{2}\right)$ e ciclofosfamida $\left(500 \mathrm{mg} / \mathrm{m}^{2}\right)($ FEC 50$)$.

Foram utilizados como critérios de exclusão pacientes acima de 70 anos de idade, portadoras de carcinoma da mama bilateral sincrônico ou assincrônico, com tumor maligno de qualquer outra localização, no ciclo grávido-puerperal, que foram submetidas à biópsia excisional do tumor, que foram inicialmente submetidas ao tratamento loco-regional cirúrgico e/ou radioterápico ou hormonioterapia, com cardiopatia grave e que não tivessem todos os dados necessários no prontuário.

As variáveis independentes estudadas foram: o estádio clínico da doença (classificado pelo sistema TNM, adotado pela UICC ${ }^{6}$ ); a idade (calculada em anos completos de vida, desde a data do nascimento até a data do diagnóstico); o estado menopausal (classificado em duas categorias: pré-menopausa e pós-menopausa; foram consideradas pós-menopausa as pacientes que relataram amenorréia por mais de 12 meses no momento do diagnóstico ${ }^{7}$ ); a graduação histológica (os tumores foram graduados histologicamente de acordo com o método de Bloom-Richardson ${ }^{8}$ ); o estado linfonodal axilar (envolvimento ou não dos linfonodos regionais, segundo a classificação pela UICC ${ }^{6}$ ). Também estudamos o volume do tumor, que foi obtido pela fórmula: volu$m e=\left(d^{3} \times \pi\right) 6$, na qual $d$ é a média da soma dos dois maiores diâmetros da lesão9. Este volume foi avaliado em dois momentos distintos, utilizando-se sistema métrico $\left(\mathrm{cm}^{3}\right)$ em um primeiro momento, previamente à quimioterapia neoadjuvante, e, posteriormente, após 21 dias do último ciclo da quimioterapia neoadjuvante. Outra variável independente estudada foi o número de ciclos aplicados na neoadjuvância, que foram divididos em duas categorias: três ciclos e quatro ciclos. Cada ciclo foi administrado em intervalo mínimo de 21 dias, via intravenosa, com realização prévia de hemograma completo. Foram considerados parâmetros aceitáveis para a sua aplicação: hemoglobina $>10 \mathrm{~g} / \mathrm{dL}$, leucócitos $>3.000 / \mathrm{mL}$ e plaquetas $>100.000 / \mathrm{mL}$.

A variável dependente estudada foi a resposta tumoral. Para o cálculo da resposta tumoral à quimioterapia foi utilizado o critério preconizado pela UICC, em que se comparam os produtos dos valores inicial e final do tumor ${ }^{10}$. Foram consideradas como medidas clínicas inicial e final o diâmetro médio do tumor ao exame clínico utilizando-se uma régua ou paquímetro e registrado no sistema métrico (centímetros) e sua medida utilizada para calcular o volume tumoral. Como medida final, no exame histopatológico, foi usado o diâmetro médio à macroscopia da peça cirúrgica.

Foi considerada resposta clínica completa a ausência de massa tumoral palpável ao exame clínico e resposta patológica completa a ausência de qualquer evidência macroscópica de tumor à inspeção da peça cirúrgica, sem levar em conta os achados na microscopia ${ }^{11}$. A resposta clínica foi então classificada como: completa (na ausência de tumor mensurável), parcial (redução entre 50 e 100\%), estacionária (redução menor que $50 \%$ ou aumento menor que $25 \%$ ) ou progressão (aumento maior que 25\% ou ocorreu o aparecimento de novas lesões). Para a análise dos dados as respostas clínicas foram agrupadas ainda em duas categorias: as pacientes com resposta objetiva, ou seja, aquelas com resposta clínica completa ou parcial, e aquelas sem resposta objetiva, ou seja, nas quais se observou resposta estacionária ou progressão.

$\mathrm{Na}$ análise estatística, para o estudo da associação entre as variáveis (idade, estado menopausal, volume tumoral pré-quimioterapia, estado axilar, estádio, esquema terapêutico e número de ciclos) foram utilizados os testes do $\chi 2$ e o exato de Fisher. Para as variáveis quantitativas (volume tumoral do anátomo patológico e volume tumoral clínico pós-quimioterapia) foi utilizado o coeficiente de correlação de Pearson. Foram calculadas medidas de tendência central e variabilidade para as variáveis quantitativas e os resultados foram apresentados em forma de gráficos. O nível de significância utilizado foi de $5 \% 12$.

Este trabalho foi aprovado pelo Comitê de Ética da Faculdade de Medicina de Botucatu em 2 de agosto de 2004.

\section{Resultados}

$\mathrm{O}$ volume do tumor antes da quimioterapia neoadjuvante variou de $2,5 \mathrm{~cm}^{3}$ a $1767,0 \mathrm{~cm}^{3}$, com média de $150,1 \mathrm{~cm}^{3}$. Um volume menor que $65,4 \mathrm{~cm}^{3}$, que 
corresponde a um tumor de diâmetro médio menor que $5 \mathrm{~cm}$, foi observado em $59,8 \%$ das pacientes, e maior ou igual a $65,4 \mathrm{~cm}^{3}$, em 40,2\% .

Em relação às características clínicas e anátomopatológicas das pacientes submetidas à quimioterapia primária, observamos que a maioria das mulheres $(58,8 \%)$ se encontrava com mais de 50 anos e $53,6 \%$ estavam na pós-menopausa. A idade da população estudada variou de 30 a 73 anos, com média 52,2 anos, e a raça branca foi a mais freqüente, com $91,8 \%$. Em relação ao grau histológico, o grau 1 esteve presente em 21,5\% das vezes, o grau 3 em $32,1 \%$ e o grau mais freqüente foi o 2, encontrado em $46,4 \%$ dos casos. Quanto ao comprometimento axilar das pacientes, 46,4\% delas apresentavam axila negativa e 53,6\% encontravam-se com axila comprometida. Sobre o estádio clínico, observamos $37,1 \%$ no estádio IIA, 19,7\% no IIB, 21,6\% no IIIA e $21,6 \%$ no IIIB.

A distribuição dos esquemas terapêuticos foi muito semelhante, $49,5 \%$ das pacientes receberam AC e 50,5\% receberam FEC. Em 61,8\% das vezes, elas receberam três ciclos e, em $38,2 \%$, quatro ciclos de quimioterapia neoadjuvante.
Observamos que, após a quimioterapia primária, o comprometimento axilar diminuiu de $53,6 \%$ para $32 \%$ e as pacientes com axila livre passaram de 46,4 para $68 \%$.

A Tabela 1 mostra a distribuição das pacientes segundo a resposta clínica objetiva à quimioterapia primária, isto é, resposta clínica maior ou igual a $50 \%$, relacionada com a idade, o estado menopausal, o volume do tumor, o comprometimento axilar, o estádio, o esquema terapêutico e o número de ciclos de quimioterapia primária.

Observamos resposta clínica objetiva à quimioterapia primária em $64,9 \%$ das pacientes. Nas pacientes menopausadas, esta resposta foi de $67,3 \%$ em comparação com $62,2 \%$ de resposta das pacientes na pré-menopausa. Quanto ao volume tumoral, as pacientes com tumores menores que $65,4 \mathrm{~cm}^{3}$ (diâmetro menor $5 \mathrm{~cm}$ ) apresentaram $67,2 \%$ de resposta clínica objetiva, comparado a $61,2 \%$ de resposta das pacientes com tumores maiores que $65,4 \mathrm{~cm}^{3}$. Nas pacientes com axila negativa, observou-se menor resposta em relação àquelas com axilas positivas, respectivamente 47 e $57,1 \%$. Em relação aos estádios II e III, obtivemos

Tabela 1 - Distribuição das pacientes segundo a resposta clínica objetiva à quimioterapia primária.

\begin{tabular}{|c|c|c|c|c|c|}
\hline & \multicolumn{2}{|c|}{ Presente } & \multicolumn{2}{|c|}{ Ausente } & \multirow{2}{*}{$\begin{array}{c}\text { Valor } \\
\mathbf{P} \\
\end{array}$} \\
\hline & n & (\%) & n & $(\%)$ & \\
\hline Idade & & & & & 1,000 \\
\hline Menos de 50 anos & 26 & 65,0 & 20 & 35 & \\
\hline 50 anos ou mais & 37 & 64,9 & 14 & 35,1 & \\
\hline Menopausa & & & & & 0,795 \\
\hline Pré-menopausa & 28 & 62,2 & 17 & 37,8 & \\
\hline Pós-menopausa & 35 & 67,3 & 17 & 32,7 & \\
\hline Volume tumoral (pré-tratamento) & & & & & 0,719 \\
\hline Menor $65,4 \mathrm{~cm}^{3}$ & 39 & 67,2 & 19 & 32,8 & \\
\hline Maior $65,4 \mathrm{~cm}^{3}$ & 24 & 61,5 & 15 & 38,5 & \\
\hline Axila (avaliação clínica pré-tratamento) & & & & & 0,461 \\
\hline Negativa & 16 & 47,0 & 18 & 53,0 & \\
\hline Positiva & 36 & 57,1 & 27 & 42,8 & \\
\hline Estádio & & & & & 0,275 \\
\hline ॥ & 34 & 61,8 & 13 & 38,2 & \\
\hline III & 29 & 69,0 & 29 & 31,0 & \\
\hline Esquema terapêutico & & & & & 0,573 \\
\hline AC & 33 & 68,7 & 15 & 31,3 & \\
\hline FEC50 & 30 & 61,2 & 19 & 38,8 & \\
\hline Número de ciclos & & & & & 0,0354 \\
\hline 3 & 33 & 55 & 27 & 45,0 & \\
\hline 4 & 29 & 79,1 & 8 & 18,9 & \\
\hline
\end{tabular}


respostas clínicas objetivas respectivamente de 61,8 e $69 \%$. As pacientes que receberam doxorubicina e ciclofosfamida apresentaram resposta clínica objetiva de $68,1 \%$ comparado a $61,2 \%$ de resposta daquelas que receberam 5 -fluorouracil, epirubicina e ciclofosfamida. A única variável que apresentou diferença significante foi o número de ciclos, pois observamos $55 \%$ de resposta nas pacientes que receberam três ciclos em comparação com $79,1 \%$ de resposta daquelas que receberam quatro ciclos.

Na Tabela 2 observamos a distribuição das pacientes segundo a resposta clínica completa e patológica completa à quimioterapia primária, relacionando com as mesmas variáveis citadas acima.

A resposta clínica completa à quimioterapia primária foi observada em 12,3\% das pacientes. A única variável que foi estatisticamente significante foi o número de ciclos. Observou-se que as pacientes que receberam três ciclos apresentaram $10 \%$ de resposta clínica completa em comparação com $16,2 \%$ de resposta daquelas que receberam quatro ciclos.
A resposta patológica completa à quimioterapia primária foi observada em $10,3 \%$ das pacientes e nenhuma variável apresentou diferença significativa em relação a este parâmetro. Em relação à idade, observamos maior taxa de resposta patológica completa nas pacientes com menos de 50 anos $(14,6 \%)$, comparada àquelas maiores ou iguais a 50 anos $(5,3 \%)$. Já em relação ao volume tumoral, observamos $67,2 \%$ de resposta clínica completa e $9,4 \%$ de resposta patológica completa nos tumores menores que 65,4 $\mathrm{cm}^{3}(<5 \mathrm{~cm})$, em relação a $61,5 \%$ de resposta clínica completa e $10,5 \%$ de resposta patológica completa dos tumores com volumes maiores que $65,4 \mathrm{~cm}^{3}$. Quanto ao comprometimento axilar, observamos uma resposta patológica completa de $6,7 \%$ nas pacientes com axilas negativas comparada a $11,5 \%$ naquelas com axilas comprometidas. As pacientes que receberam AC tiveram $10,4 \%$ de resposta clínica completa e 6,2\% de resposta patológica completa, em comparação a $14,3 \%$ de resposta clínica e patológica completas entre as pacientes que receberam FEC50.

Tabela 2 - Distribuição das pacientes segundo a resposta clínica completa e patológica completa à quimioterapia primária

\begin{tabular}{|c|c|c|c|c|c|c|c|c|c|c|}
\hline \multirow{3}{*}{ Completa } & \multicolumn{5}{|c|}{ Resposta clínica completa à quimioterapia primária } & \multicolumn{5}{|c|}{ Resposta patológica completa à quimioterapia primária } \\
\hline & \multicolumn{2}{|c|}{ Presente } & \multicolumn{2}{|c|}{ Ausente } & \multirow[b]{2}{*}{$P$} & \multicolumn{2}{|c|}{ Presente } & \multicolumn{2}{|c|}{ Ausente } & \multirow[b]{2}{*}{$\mathrm{p}$} \\
\hline & n & $\%$ & n & $\%$ & & n & $\%$ & n & $\%$ & \\
\hline Idade & & & & & 0,825 & & & & & 0,104 \\
\hline$<50$ anos & 10,0 & 36 & 90,0 & & 6 & 14,6 & & 34 & 85,4 & \\
\hline$>50$ anos & 8 & 14,0 & 49 & 86,0 & & 3 & 5,3 & 54 & 94,7 & \\
\hline Menopausa & & & & & 0,795 & & & & & 0,795 \\
\hline pré-menopausa & 6 & 13,3 & 39 & 86,7 & & 7 & 15,2 & 38 & 84,8 & \\
\hline pós-menopausa & 6 & 11,5 & 46 & 88,5 & & 2 & 3,9 & 50 & 96,1 & \\
\hline Volume fumoral (pré-tratamento) & & & & & 0,814 & & & & & 0,732 \\
\hline menor $65,4 \mathrm{~cm}^{3}$ & 7 & 12 & 51 & 88 & & 5 & 9,4 & 53 & 90,6 & \\
\hline maior $65,4 \mathrm{~cm}^{3}$ & 5 & 12,9 & 34 & 87,1 & & 4 & 10,5 & 34 & 89,5 & \\
\hline Axila (avaliação clínica pré-tratamento) & & & & & 0,547 & & & & & 0,498 \\
\hline negativa & 6 & 13,3 & 39 & 86,7 & & 3 & 6,7 & 42 & 97,3 & \\
\hline positiva & 6 & 11,4 & 46 & 88,6 & & 6 & 11,5 & 46 & 88,5 & \\
\hline Estádio & & & & & 0,725 & & & & & 0,730 \\
\hline II & 6 & 10,9 & 49 & 89,1 & & 6 & 10,9 & 49 & 89,1 & \\
\hline III & 6 & 14,2 & 36 & 85,8 & & 3 & 7,3 & 39 & 92,7 & \\
\hline Esquema terapêutico & & & & & 0,526 & & & & & 0,296 \\
\hline$A C$ & 5 & 10,4 & 43 & 89,6 & & 3 & 6,2 & 45 & 93,2 & \\
\hline FEC50 & 7 & 14,3 & 42 & 85,7 & & 7 & 14,3 & 42 & 85,7 & \\
\hline Número de ciclos & & & & & 0,0320 & & & & & 1,000 \\
\hline 3 & 6 & 10,0 & 54 & 90,0 & & 5 & 8,4 & 55 & 91,6 & \\
\hline 4 & 6 & 16,2 & 31 & 83,8 & & 4 & 10,8 & 33 & 89,2 & \\
\hline
\end{tabular}


A Figura 1 mostra o diagrama de dispersão relativo ao volume tumoral após a quimioterapia primária e ao volume tumoral do anátomo-patológico. Observamos correlação significante entre o volume tumoral mensurado clinicamente após a quimioterapia primária e o volume mensurado pelo exame anátomo-patológico.

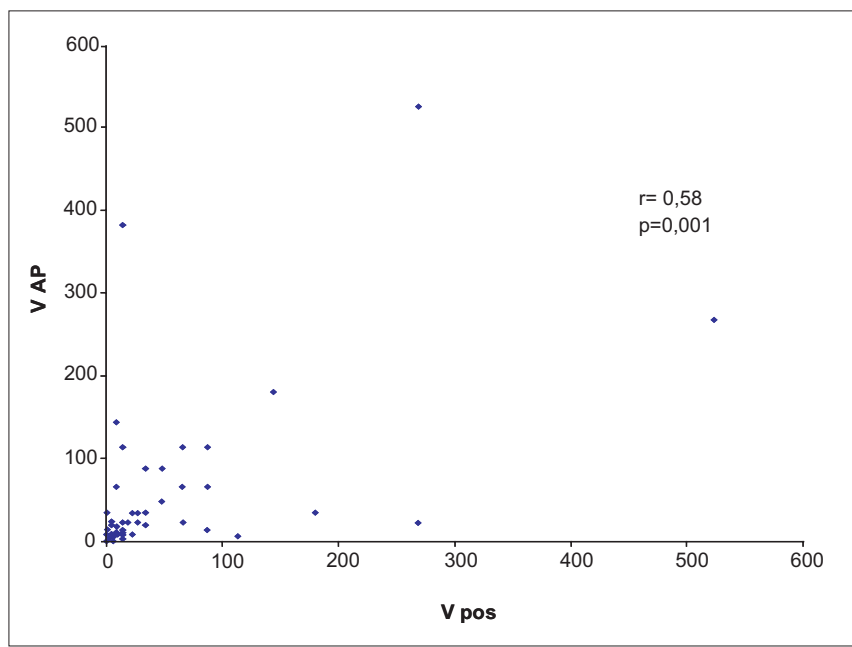

Figura 1 - Diagrama de dispersão relativo ao volume tumoral após a quimioterapia primária (V pós) e ao volume tumoral do anátomopatológico (V AP)

\section{Discussão}

O objetivo do nosso estudo foi avaliar a resposta à quimioterapia primária nas pacientes com câncer de mama estádios II e III. Em revisão da literatura com 18 grandes estudos sobre quimioterapia neoadjuvante, foram observadas respostas clínicas completas, variando de 3 a $52 \%$, e respostas patológicas completas, que oscilaram de 4 a $31 \%$. No entanto, estes estudos utilizaram esquemas diferentes com taxanes, antracíclicos e número de ciclos pré-operatórios distintos ${ }^{1}$. Dois trabalhos relevantes são importantes para comparação com nosso estudo: NSAPB-18 ${ }^{2}$, que indicou $36 \%$ de resposta clínica completa e $13 \%$ de resposta patológica completa utilizando quatro ciclos de quimioterapia pré-operatória com AC somente, e o NSABP- $27^{3}$, que obteve $45,4 \%$ de resposta clínica parcial, 40,2\% de resposta clínica completa e aproximadamente $10 \%$ de resposta patológica completa, também com o mesmo esquema terapêutico (AC) em um dos braços do estudo. Um terceiro estudo relevante para comparação com nosso trabalho é o EORTC10902 ${ }^{13}$, que utilizou o esquema de quatro ciclos de quimioterapia primária com FEC 50. Este estudo mostrou uma taxa de $49 \%$ de resposta clínica objetiva, $7 \%$ de resposta clínica completa e $4 \%$ de resposta patológica completa. Nosso trabalho mostrou que das 97 pacientes submetidas à quimioterapia neoadjuvante, $64,9 \%$ obtiveram resposta clínica objetiva à quimioterapia primária. A taxa de resposta clínica completa foi de $12,3 \%$ e de resposta patológica completa de $10,3 \%$, mostrando resultados semelhantes com os dados destes três estudos citados, os quais aplicaram as mesmas drogas e número de ciclos semelhantes ao nosso, porém com número muito superior de pacientes.

Em relação aos fatores clínicos e patológicos relacionados com a resposta tumoral, abordamos a idade, o estado menopausal, o comprometimento axilar e o estádio da doença. Para o tratamento neoadjuvante, a idade e o estado menopausal não têm seu papel definido como fatores preditivos relacionados com a resposta loco-regional à quimioterapia neoadjuvante. Estudos como o de GEPARTRIO ${ }^{14}$ e de Dieras et al. ${ }^{15}$, embora utilizassem o esquema AC acrescido de taxane, não encontraram relação destes parâmetros com a resposta ao tratamento primário. Porém, o estudo NSABPB-27 mostra resposta patológica completa em porcentagem maior $(14,7 \%)$ nas mulheres com menos de 50 anos, comparado com as mulheres com mais de 50 anos $(12,4 \%)^{3}$. Apesar de os nossos resultados apontarem maior resposta nas pacientes com menos de 50 anos $(14,6 \%)$ em comparação àquelas com 50 anos ou mais $(5,3 \%)$, semelhante aos achados do NSABP-27, nossos resultados não tiveram correlação significante. Também não identificamos relação entre a idade e o estado menopausal com a resposta clínica loco-regional à quimioterapia primária.

Quanto ao tamanho do tumor, este constitui um dos fatores prognósticos de maior importância. Há controvérsia na literatura quanto à relação entre o tamanho tumoral e a resposta à quimioterapia primária. Bonadonna et al. ${ }^{16}$, em artigo de revisão sobre séries de esquemas de drogas em neoadjuvância, mostraram que tumores menores respondem melhor à quimioterapia primária do que tumores maiores. O mesmo foi observado no estudo NSABPB-27, no qual tumores maiores que $4 \mathrm{~cm}$ tiveram resposta patológica de $13,1 \%$ comparada com resposta de $17,4 \%$ dos tumores menores $^{3}$. Nossos resultados, como já mencionado pela semelhança em um dos braços do estudo NSABPB-27, não apontaram correlação significante entre a resposta clínica loco-regional e patológica completa e o volume tumoral inicial, apesar da tendência de melhor resposta dos tumores com volumes menores que $65,4 \mathrm{~cm}^{3}$ (diâmetro médio $5 \mathrm{~cm}$ ).

O comprometimento linfonodal axilar constitui o melhor fator prognóstico de sobrevida livre da doença em mulheres com carcinoma de mama ${ }^{17}$. No entanto, 
o papel do estado linfonodal axilar pré-quimioterapia como fator preditivo de resposta loco-regional não está claro. Alguns estudos utilizando esquemas de associação de antracíclicos e taxanes não mostraram relação do acometimento axilar com a resposta à quimioterapia primária ${ }^{14,15}$. Inversamente, o estudo NSABPB-18 mostrou que o estado da axila parece constituir fator preditivo de resposta clínica completa à quimioterapia primária ${ }^{2}$, principalmente em tumores de pequeno tamanho. No estudo NSABP-27 também se encontrou correlação entre o estado axilar e a resposta: $16,3 \%$ de resposta patológica completa nas pacientes axilas positivas, comparado com $12,5 \%$ de resposta nas axilas negativas ${ }^{3}$. Não encontramos correlação significante entre o estado linfonodal axilar inicial e a resposta clínica loco-regional à quimioterapia, porém notamos uma tendência de melhor resposta nas pacientes axilas positivas. Observamos $11,5 \%$ de resposta patológica completa nas pacientes axilas positivas, comparado com $6,7 \%$ de resposta nas axilas negativas.

A comparação entre o número de ciclos de quimioterapia primária é difícil. Na literatura, diferentes esquemas são utilizados, mostrando resultados heterogêneos e dificultando as análises. Atualmente, há tendência à utilização de período maior de tratamento neoadjuvante nas pacientes com perfil mais desfavorável à terapêutica, visando aumentar as taxas de resposta objetiva e, com isso, a chance de cirurgia conservadora ${ }^{18}$. Outros estudos recentes indicam que número maior de ciclos promoveria maior número de respostas clínicas e patológicas completas ${ }^{2,19,20}$. Obtivemos resultados significativamente melhores quando comparamos a resposta clínica objetiva e a resposta clínica completa com número de ciclos recebidos (três ou quatro). Observamos uma resposta clínica objetiva em 79,1\% das pacientes submetidas a quatro ciclos de quimioterapia primária, comparada com $55 \%$ de resposta clínica objetiva entre as que receberam três ciclos apenas. As pacientes submetidas a quatro ciclos tiveram $16,2 \%$ de respostas clínicas completas, comparado a $10,0 \%$ daquelas que receberam três ciclos. Houve, também, tendência de resposta patológica completa mais freqüente entre as pacientes que receberam quatro ciclos $(10,8 \%)$, comparada às que receberam três ciclos $(8,4 \%)$. Os nossos dados dão suporte à tendência atual de prolongar o tratamento quimioterápico primário para obter a maior resposta possível.

Para determinarmos qual esquema é mais eficaz entre FEC50 e AC, identificamos dois estudos de maior relevância na literatura, os quais utilizaram esses esquemas e pacientes em estádios semelhantes aos nossos casos. O estudo EORTC10902 mostrou, como já citado, uma taxa de $49 \%$ resposta clínica objetiva, $7 \%$ de resposta clínica completa e $4 \%$ de resposta patológica completa utilizando quatro ciclos de FEC neoadjuvante ${ }^{13}$, e o NSABP-18 indicou $80 \%$ de resposta clínica objetiva, $36 \%$ de resposta clínica completa e 9\% de resposta patológica completa, utilizando-se quatro ciclos de $\mathrm{AC}$ neoadjuvante ${ }^{2}$. A análise destes dois trabalhos indica uma melhor resposta quando o esquema AC é utilizado. Nosso estudo também aponta para uma melhor resposta objetiva quando utilizamos o AC $(68,7 \%)$ em relação ao $\operatorname{FEC50}(61,3 \%)$. Porém, quando comparamos a resposta clínica completa e patológica completa, temos um melhor resultado com o uso do FEC50 (14,3 e 14,3\%) comparado com AC $(10,4$ e $8,4 \%)$. O número pequeno de casos estudados pode explicar o achado não concordante com estes dois trabalhos citados.

Em estudo prospectivo no qual se compararam dois grupos de pacientes com 30 mulheres em cada, com câncer de mama estádio IIIA, o primeiro grupo recebeu tratamento com FEC50 (o mesmo do nosso trabalho) e obteve-se resposta clínica objetiva de $93 \%$, resposta clínica completa de $13 \%$ e patológica completa também de $13 \%$. Nosso trabalho se correlaciona melhor com estes dados, possivelmente pela maior semelhança entre as populações estudadas.

Outro ponto importante é avaliar a resposta à quimioterapia primária para determinar a real dimensão do tumor residual, pois um dos objetivos do tratamento neoadjuvante é a cirurgia conservadora. Além disso, vários trabalhos apontam maior período livre de doença e taxas maiores de sobrevida global nas pacientes com resposta patológica completa ${ }^{1-3}$. Assim, tenta-se encontrar o melhor método de avaliar a resposta ao tratamento sistêmico primário. Em alguns estudos houve a comparação do exame clínico com a mamografia e mostrou-se que o primeiro tem uma acurácia maior que o segundo para avaliação da resposta à quimioterapia primária, respectivamente de 92 a $94 \%$ comparado com 78 a 78,9\% ${ }^{21,22}$. Porém, para o grupo do hospital MD Anderson houve acurácia de $66 \%$ para o exame físico comparado a $70 \%$ com a mamografia ${ }^{23}$. Outros métodos novos estão sendo empregados, como a ultra-sonografia e a ressonância nuclear magnética, porém não há consenso sobre qual o melhor método. Nosso trabalho procurou estudar apenas a correlação da resposta à quimioterapia primária avaliada pelo exame clínico com o achado anátomo-patológico. Observa-se uma concordância significante, mostrando, assim, que o 
exame clínico pode ser usado para avaliar a resposta ao tratamento quimioterápico primário.

A quimioterapia primária proporciona oportunidade de entender melhor o comportamento do câncer mamário, permitindo o estudo de fatores que influenciam a resposta tumoral ao quimioterápico. Há uma série de dados contraditórios na literatura mundial, diferentes esquemas terapêuticos estudados em populações bem distintas, dificultando a interpretação dos estudos e a comparação com o nosso estudo. Os trabalhos mais recentes sobre quimioterapia primária mostram respostas superiores com o uso de drogas como taxanes e transtuzumab do que as obtidas com os antracíclicos. Em nosso país, as pacientes portadoras de câncer mamário tratadas pelo SUS ficam, na maioria das vezes, restritas a drogas estudadas neste trabalho. Soma-se o fato de as mulheres do nosso país possuírem características físicas e biológicas que lhe são peculiares. Isto reflete o motivo da maior concordância dos nossos dados com os da literatura brasileira do que com os da literatura internacional. Nosso estudo, desta maneira, contribuiu para melhor compreensão do comportamento biológico do câncer de mama nas pacientes brasileiras frente ao tratamento quimioterápico primário. Analisamos os fatores clínicos, patológicos e comparamos dois diferentes esquemas terapêuticos que são acessíveis à grande maioria das nossas pacientes com câncer de mama, o que é inédito na literatura.

\section{Referências}

1. Trudeau M, Sinclair SE, Clemons M; Breast Cancer Disease Site Group. Neoadjuvant taxanes in the treatment of non-metastatic breast cancer: a systematic review. Cancer Treat Rev. 2005; $31(4): 283-302$.

2. Wolmark N, Wang J, Mamounas E, Bryant J, Fisher B. Preoperative chemotherapy in patients with operable breast cancer: nine-year results from National Surgical Adjuvant Breast and Bowel Project B-1 8. J Natl Cancer Inst Monogr. 2001; (30):96-102.

3. Bear HD, Anderson S, Brown A, Smith R, Mamounas E, Fisher B, et al. The effect on tumor response of adding sequential preoperative docetaxel to preoperative doxorubicin and cyclophosphamide: preliminary results from National Surgical Adjuvant Breast and Bowel Project Protocol B-27. J Clin Oncol. 2003; 21 (22):4165-74.

4. Budzar AU, Ibrahim NK, Francis D, Booser DJ, Thomas ES, Theriault $\mathrm{RL}$, et al. Significantly higher pathologic complete remission rate after neoadjuvant therapy with trastuzumab, paclitaxel, and epirubicin chemotherapy: results of a randomized trial in human epidermal growth factor receptor 2-positive operable breast cancer. J Clin Oncol. 2005; 23(16):3676-85.

5. Instituto Nacional de Câncer - INCA [sítio na Internet]. Incidência de câncer no Brasil. 2005 [citado 2005 Maio 6 ]. Disponível em: http://www.inca.gov.br/estimativa/2005/

6. Greene FL, Page DL, Fleming ID, Fritz A, Balch CM, Haller DG, et al., editors. AJCC cancer staging manual. $6^{\text {th }}$ ed. New York: Springer; 2002.

7. World Health Organization. Research of the menopause. Geneva: WHO; 1981. p. 8-10. [Technical Report, 670].

8. Bloom HJ, Richardson WW. Histological grading and prognosis in breast cancer; a study of 1409 cases of which 359 have been followed for 15 years. Br J Cancer. 1957; 11 (3):359-77.

9. Dixon JM, Renshaw L, Bellamy C, Stuart M, Hoctin-Boes G, Miller WR. The effects of neoadjuvant anastrozole (Arimidex) on tumor volume in postmenopausal women with breast cancer: a randomized, double-blind, single-center study. Clin Cancer Res. 2000; 6(6): 2229-35.

10. Hayward JL, Rubens RD, Carbone PP, Heuson JC, Kumaoka S, Segaloff A. Assessment of response to therapy in advanced breast cancer. A project of the programme on clinical oncology of the International Union against Cancer, Geneva, Switzerland. Eur J Cancer. 1978; 14(1 1):1291-2.

11. Feldman LD, Hortobagyi GN, Buzdar AU, Ames FC, Blumenschein GR. Pathological assessment of response to induction chemotherapy in breast cancer. Cancer Res. 1986; 46(5):2578-81.

12. Fisher LD, Van Belle G. Biostatistics: a methodology for the health sciences. New York: Wiley; cl993.

13. van der Hage JA, van de Velde CJ, Julien JP, Tubiana-Hulin M, Vandervelden C, Duchateau L. Preoperative chemotherapy in primary operable breast cancer: results from the European Organization for Research and Treatment of Cancer Trial 10902. J Clin Oncol. 2001 ; 19(22):4224-37.

14. von Minckwitz G, Blohmer JU, Raab G, Lohr A, Gerber B, Heinrich $G$, et al. In vivo chemosensitivity-adapted preoperative chemotherapy in patients with early-stage breast cancer: the GEPARTRIO pilot study. Ann Oncol. 2005; 16(1):56-63.

15. Dieras V, Fumoleau $P$, Romieu $G$, Tubiana-Hulin $M$, Namer $M$ Mauriac $L$, et al. Randomized parallel study of doxorubicin plus paclitaxel and doxorubicin plus cyclophosphamide as neoaduvant treatment os patients with breast cancer. J Clin Oncol. 2004; 22(24):4958-65.

16. Bonadonna G, Valagussa P, Zucali R, Salvadori B. Primary chemotherapy in surgically resectable breast cancer. CA Cancer J Clin. 1995; 45(4):227-43. Review.

17. Cutuli B, Velten M, Martin C. Assessment of axillary lymph node involvement in small breast cancer: analysis of 893 cases. Clin Breast Cancer. 2001; 2(1):59-65; discussion 66 .

18. Mano MS, Awada A. Primary chemotherapy for breast cancer: the evidence and the future. Ann Oncol. 2004; 15(8):1161-71.

19. Dueñas Rodríguez $B$, Jiménez Armenteros FM, Gómez Ortega A, Martínez Gallego G, Sánchez Rovira P, Medina Cuadros M. Tratamiento conservador en los carcinomas avanzados de mama. Bol GEICAM. 2004; (12): 14-8.

20. Estevez LG. Neoadjuvant chemotherapy in breast cancer. Rev Oncol. 2004; 6(5):314-20. 
21. El-Didi MH, Moneer MM, Khaled HM, Makarem S. Pathological assessment of the response of locally advanced breast cancer to neoadjuvant chemotherapy and its implications for surgical management. Surg Today. 2000; 30(3): 249-54.

22. Moneer $M$, El-Didi $M$, Khaled $H$. Breast conservative surgery: is it appropriate for locally advanced breast cancer following downstaging by neoadjuvant chemotherapy? A pathological assessment. Breast. 1999; 8(6):315-9.

23. Chagpar AB, Middleton LP, Sahin AA, Dempsey P, Buzdar AU, Mirza AN, et al. Accuracy physical examination, ultrasonography, and mammography in predicting residual pathologic tumor size in patients treated with neoadjuvant chemotherapy. Ann Surg. 2006; 243(2):257-64. 